\title{
The study of correlation between forward head posture and shoulder pain: A STROBE-compliant cross-sectional study
}

\author{
Hyun-Joong Kim ${ }^{a}{ }^{\circledR}$, DongJin Lee ${ }^{b}$ \\ aSports Rehabilitation Center, The Better Hospital, Gwangju, Republic of Korea \\ ${ }^{b}$ Department of Physical Therapy, Gwangju Health University, Gwangju, Republic of Korea
}

Objective: The forward head posture (FHP) is strongly related to the rounded shoulder posture (RSP), which is associated with shoulder pain.

Design: Observational cross sectional study design

Methods: A total of 37 were enrolled in the study, 22 individuals with FHP(experimental group) and 15 healthy adults(control group). Correlation with differences between groups was analysed through craniovertebral angle (CVA) representing FHP for both groups, neck disability index (NDI) indicating neck pain, disability of the arm, shoulder and hand (DASH) indicating shoulder pain.

Results: There was a significant difference in the results of CVA, NDI, and DASH in FHP and healthy adults ( $p<0.05$ ). Significant correlations were found between DASH and CVA in FHP participants $(r=-0.656, p=0.001)$. Also, in the regression analysis results of DASH and CVA, the regression model was found to be suitable and the variation in DASH could be explained by $43 \%(\mathrm{~F}=15.118, \mathrm{p}=0.001)$.

Conclusions: Shoulder pain and neck discomfort are potentially related, and an increase in shoulder pain can increase FHP.

Key Words: Forward head posture, Rounded shoulder posture, Shoulder pain, Neck pain

\section{Introduction}

Posture is the alignment of body parts and is an important health indicator. It should be matched with a specific body position in the space that minimizes antigravity stress on body tissues [1]. Inappropriate posture consists of incorrect interrelationships between each body segment. This causes muscle tension and shortening, making it difficult to achieve proper joint motion and can cause pain [2].

The forward head posture (FHP) has been shown to be a general posture displacement, and the typical estimate is about $66 \%$ of the patient population. In general, these abnormal postures are associated with the development and persistence of many disorders, including cervicogenic headache and migraine, myofascial pain syndrome, abnormal shoulder blade movement, and temporomandibular joint disorder [3].

FHP is an anterior deviation of the head accompanied by hyper-extension of the neck, which is the upper trapezius, splenius capitis, semispinalis capitis, and cervical erector spinae. It is associated with shortening of the levator scapulae $[4,5]$. If the cervical spine is neck extension for a long time, it can lead to FHP [6-8]. If the head is not aligned with the vertical axis of the body, malalignment occurs as in the rounded shoulder posture (RSP) [9]. RSP is the anterior deviation of the shoulder associated with the protracted position of the scapula due to the muscle imbalance between the shortened pectoralis minor and 


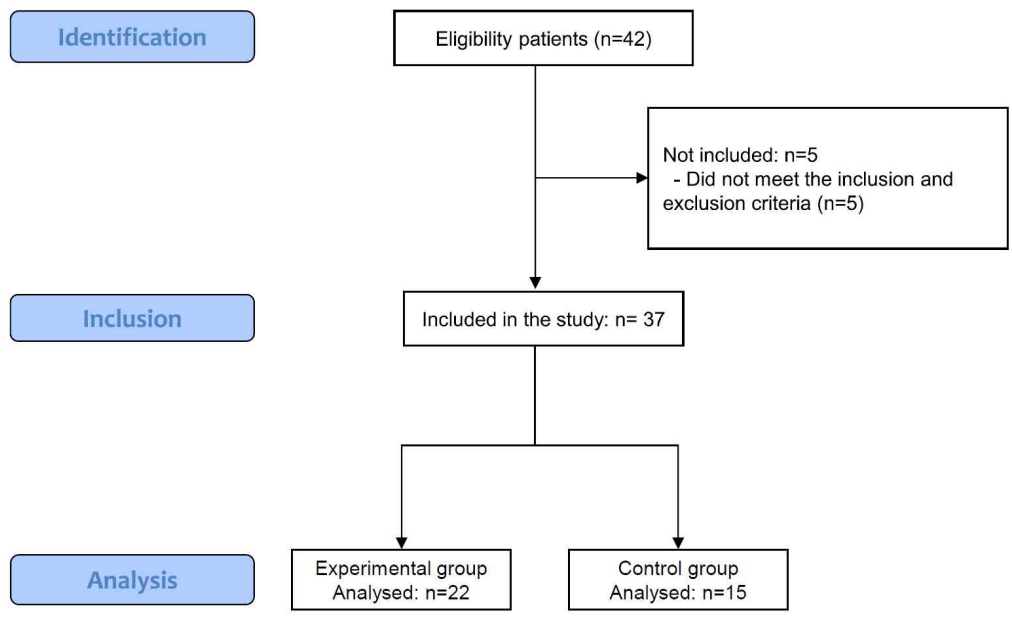

Figure 1. STROBE flowchart for patient recruitment

the elongated middle trapezius [5].

Kwonet al [10] concluded that the altered head position changes the kinematics of the head and shoulders and the activation of muscles. In addition, a study by Thigpen et al [11] reported that the internal rotation of the shoulder complex was significantly higher in patients with RSP and THP of the shoulder. Considering the relationship between neck posture and shoulder pain $[8,12]$, an in-depth understanding of the effect on the scapular is required, which can be effective in finding strategies for preventing or reducing shoulder pain.

Despite the importance of the problem caused by the change in posture, research on the direct correlation between the posture of the head and neck and the shoulder is insufficient. Therefore, FHP due to an imbalance in the muscles around the neck may be related to shoulder pain. This study aims to investigate the correlation in detail through the difference between the head spine angle, neck pain, and shoulder pain between participants with FHP and healthy adults.

\section{Methods}

\section{Study design}

The design of this study was conducted in accordance with the Strengthening the Reporting of Observational Studies in Epidemiology Statement (STROBE) guidelines as an observational cross-sectional study [13].

\section{Participants}

The inclusion of potential participants for this study was recruited through the university bulletin board of Gwangju Health University in Gwangju, South Korea. Forty-two potential participants were recruited, and 37 participants were finally enrolled in the study.

The study procedure is shown in Figure 1 of the STROBE flow chart. The inclusion criteria and exclusion criteria are as follows [14]. Inclusion criteria (craniovertebral angle $[\mathrm{CVA}]<49^{\circ}$, if one perceives that the neck is curved, shoulder pain [numeric pain rating scale $>3]$ ) and exclusion criteria (a history of injury or surgery on the shoulder joint, scoliosis or kyphosis, neurological problems of the neck or shoulder, rheumatoid arthritis). The recruitment of the control group comparable to the experimental group with shoulder pain recruited healthy adults over 20 years of age.

All procedures were carried out in accordance with the Declaration of the World Medical Association and with the Declaration of Helsinki guidelines.

\section{Outcomes}

The primary outcome measure of this study is CVA. Participant sits on a chair, looking straight ahead, arms naturally on his knees, and neck in a position that keeps him most comfortable. The angle between the line connecting $\mathrm{C} 7$ and the horizontal line in the tragus of ear was measured using a goniometer 
(ICC $=0.88)$. The smaller the CVA, the more FHP progressed. According to previous studies, this study considered FHP less than $49^{\circ}[15]$.

Secondary outcome measures include Neck disability index (NDI), Disability of the arm, shoulder and hand (DASH). The NDI was designed to measure the participant's neck pain and dysfunction with a total of 10 questions [16, 17]. The NDI score is the sum of each item, and the higher the score, the greater the dysfunction related to abnormalities around the neck. The NDI interpreting scores $0-4=$ no disability, $5-14=$ mild disability, 15-24 = moderate disability, 25-34= severe disability, 35 or more $=$ complete disability. DASH was developed to measure the effects of various functionalities on musculoskeletal health and injury affecting the upper limb [18]. In Korea, this tool was applied mainly to patients with upper limb musculoskeletal disorders, and its reliability and validity were proven [19]. DASH is a questionnaire-type tool to measure the dysfunction of the upper extremity and the degree of disability in one or several areas of the upper extremity. In this study, 30 basic items and 8 optional items were used. It is a Likert scale on a scale of 1 to 5 , and it is based on the patient's daily life and movements in work/work. And the lower the score, the better.

\section{Sample size}

The sample size suitable for the study was calculated by using $\mathrm{G}^{*}$ power 3.1 (Franz Faul, Universitiat Kiel, Germany) by the difference between baseline and post-test the experiment [20] through stretching intervention among the preceding studies related to FHP. The effect size Cohen's $d=1.67$ was calculated, and when power $=0.95$ and 2 groups were set, a calculation formula was established that a total of 18 people should be assigned. More than 30 participants were required to assume the normality of dropouts due to COVID-19 and the central limit theorem, and 37 participants who signed after hearing explanations of the study according to the inclusion and exclusion criteria of the experimental group and control group were recruited.

\section{Statistical analysis}

All statistical analyses were performed using SPSS (version 25.0, IBM Corp., USA). For the homogeneity test of the experimental group and the control group, the Chi-squared test for categorical variables and the independent t-test for continuous variables were performed, and descriptive statistics were used for general characteristics. For normality test, Shapiro-Wilk test was used. Independent t-test was used to compare the difference between the two groups, Pearson's correlation coefficient was used to analyse the correlation between variables, and simple linear regression was used for the relationship between the variances. All statistical significance levels $(\alpha)$ were set to 0.05

\section{Results}

\section{General characteristics of the participants}

These are the general characteristics of the participants enrolled in this study. There was a total of 37participants in this study, and Table 1 shows the general characteristics of each group. There was no significant difference in the homogeneity test between groups $(p>0.05)$.

Table 1. General characteristics of the participants $(n=37)$

\begin{tabular}{llll}
\hline & Experimental group $(\mathrm{n}=22)$ & Control group $(\mathrm{n}=15)$ & $\chi^{2} / \mathrm{t}(\mathrm{p})$ \\
\hline Sex $($ male/female $)$ & $13 / 9$ & $10 / 5$ & $0.218(0.641)$ \\
\hline Age $($ years $)$ & $31.41(8.81)$ & $29.00(7.31)$ & $0.873(0.389)$ \\
\hline Height $(\mathrm{cm})$ & $164.64(8.06)$ & $168.73(5.91)$ & $-1.681(0.102)$ \\
\hline Weight $(\mathrm{kg})$ & $61.55(11.72)$ & $65.00(9.02)$ & $-0.962(0.343)$ \\
\hline BMI $\left(\mathrm{kg} / \mathrm{m}^{2}\right)$ & $23.19(3.46)$ & $22.74(2.26)$ & $0.435(0.666)$ \\
\hline
\end{tabular}

The values are presented mean (SD).

BMI: body mass index. 


\section{Comparison between groups analysis}

Table 2 shows the comparison between groups of CVA, NDI, and DASH evaluated in this study. There were statistically significant differences between groups $(\mathrm{p}<0.05)$.

\section{Correlation analysis}

Table 3 shows the correlations for each group of CVA, NDI, and DASH evaluated in this study. FHP participants showed significant correlations between DASH and CVA $(r=-0.656, p=0.001)$.

\section{Linear Regression analysis}

In the FHP participants, DASH and CVA showed a significant relationship with shoulder pain.The coefficient of determination $\left(\mathrm{R}^{2}\right)$ was $0.430(\mathrm{p}=0.001)$, and the regression equation was statistically significant, but the explanatory power was not high. The regression equation of the experimental group is as follows ( $y=49.612-0.133 x$ [y: CVA, $x: D A S H])$, and the CVA decreases as DASH increases with a regression coefficient of 0.133 (Table 4).

\section{Discussion}

FHP causes the musculoskeletal pain by applying a load to the cervical spine and causes malalignment such as RSP due to postural displacement. RSP changes the kinematics of the shoulder joint, causing the

Table 2. Comparison between groups $(n=37)$

\begin{tabular}{llll}
\hline & Experimental group $(\mathrm{n}=22)$ & Control group $(\mathrm{n}=15)$ & $\mathrm{t}(95 \%$ CI $)$ \\
\hline NDI (point) & $11.55(2.74)$ & $2.67(1.72)$ & $11.126^{*}(7.26,10.50)$ \\
\hline DASH (point) & $20.13(9.46)$ & $4.79(5.14)$ & $5.716^{*}(9.89,20.79)$ \\
\hline CVA (angle) & $46.94(1.92)$ & $52.21(5.08)$ & $-3.838^{*}(-8.18,-2.37)$ \\
\hline
\end{tabular}

The values are presented mean (SD).

CI: confidence interval, CVA: craniovertebral angle, DASH: disability of the arm, shoulder and hand, NDI: neck disability index.

$*_{p}<0.05$, statistically significant difference.

Table 3. The correlation between CVA, NDI and DASH $(n=22)$

\begin{tabular}{llll}
\hline Parameters & NDI & DASH & CVA \\
\hline NDI & 1 & & \\
\hline DASH & 0.137 & 1 & 1 \\
\hline CVA & -0.131 & $-0.656^{*}$ & \\
\hline
\end{tabular}

CI: confidence interval, CVA: craniovertebral angle, DASH: disability of the arm, shoulder and hand, NDI: neck disability index.

${ }^{*} \mathrm{p}<0.01$, statistically significant difference.

Table 4. Simple linear regression of DASH and CVA $(n=22)$

\begin{tabular}{llllll}
\hline & \multicolumn{2}{l}{ Unstandardized coefficients } & Standardized coefficients & \multirow{2}{*}{$\mathrm{t}$} & $\mathrm{p}$ \\
\cline { 2 - 5 } & $\mathrm{B}$ & $\mathrm{SE}$ & $\beta$ & 65.545 & \\
\hline (Constant) & 49.612 & 0.757 & -0.656 & -3.888 & $0.001^{*}$ \\
\hline DASH & -0.133 & 0.034 & & & \\
\hline
\end{tabular}

DASH: disability of the arm, shoulder and hand, $\mathrm{R}^{2}$ : coefficientofdetermination.

$\mathrm{R}^{2}=0.430, \operatorname{adj} \mathrm{R}^{2}=0.402, \mathrm{~F}=15.118(\mathrm{p}<0.01)$, Durbin-Waston $=2.109$.

${ }^{*} \mathrm{p}<0.05$, statistically significant difference. 
limitation of the range of motion and pain. Therefore, in this study, the correlation was investigated through the difference between the craniovertebral angle, neck pain, and shoulder pain between participants with FHP and healthy adults.

In this study, there were significant differences in CVA, NDI, and DASH in comparison between the experimental and control groups $(\mathrm{p}<0.05)$. This is because they were recruited through CVA, which represents FHP, and DASH, which is an assessment tool representing shoulder pain in the experimental group. In addition, a significant difference in NDI was found in previous studies on the forward head and rounded shoulder posture (FHRSP) that posture change can alter the muscle activation pattern [11]. It is believed that this is because the load was changed.

As a result of analysing the correlation in FHP, as in the hypothesis of this study, there was anintermediate effect in DASH and CVA $(\mathrm{r}=-0.656, \mathrm{p}=0.001)$. However, there was no correlation between NDI and CVA $(r=-0.131, p=0.561)$. These results may partially explain the hypothesis of the study. Motialla et al [21] reported a correlation with pain in the periscapular muscles in chronic neck pain patients $(p<0.05)$. However, in the study of Nejati et al [22], a significant positive correlation between CVA and neck pain was found $(\mathrm{p}<0.05)$ as a result that conflicted with the results of this study. This is thought to be because the comparison was made through the disability index rather than direct pain in this study.

The results of the regression analysis of DASH and CVA to examine the relevance of the FHP participants through the variables tested for normality and correlation to match the hypothesis of this study showed a significant correlation $(\mathrm{F}=15.118, \mathrm{p}=$ $0.001)$. and the variation in DASH could be explained by $43.0 \% \quad(p<0.01)$. These results were found in Khosravi et al [14], which increased winged scapula as CVA increased, and Lee et al [23] also studied the It can be said that the activity is reduced, which is partially consistent with the results of this study. However, this is different from the results of previous studies that there is no significant difference in the distance between scapula and the spine [14].

The limitation of this study is that it recruited students and employees from universities where recruitment was limited, and that by designing a single center, the age range was similar and there were many white-collar workers. In addition, there was a limitation that each variable could not be represented because no quantitative evaluation was made other than CVA. Therefore, in future studies, it can be said that it is suitable for generalization if recruitment from various institutions is expanded, and the number of participants increases. Also, it is considered that a quantitative analysis of the muscles and movements around the neck and shoulders is necessary.

\section{Conclusion}

In the comparison between the experimental group and the control group, there were significant differences in CVA, NDI, and DASH, and the relationship between the groups was found, and the relationship between the increase in DASH and CVA was identified through a linear relationship analysis. In addition, through regression analysis, it was found that DASH and CVA had a statistically significant relationship. Thus, shoulder pain and cervical spine dysfunction can be potentially related, and an increase in shoulder pain can increase FHP. It is suggested that this can be used as evidence in clinical management of patients with shoulder pain or FHP.

\section{Acknowledgements}

The Research has been conducted by the Research Grant of Gwangju Health University in 2021(2021014).

\section{Conflict of Interest}

The authors declared no potential conflicts of interestwith respect to the research, authorship, and/or publicationof this article.

\section{References}

1. Caron $\mathrm{O}$, Faure B, Brenière Y. Estimating the centre of gravity of the body on the basis of the centre of pressure in standing posture. J Biomech. 1997;30:1169-71.

2. Ruivo RM, Pezarat-Correia P, Carita A. Cervical and shoulder postural assessment of adolescents between 
15 and 17 years old and association with upper quadrant pain. Braz J Phys Ther. 2014;18:364-71.

3. Diab AA, Moustafa IM. The efficacy of forward head correction on nerve root function and pain in cervical spondylotic radiculopathy: a randomized trial. Clin Rehabil. 2012;26:351-61.

4. Falla DL, Jull GA, Hodges PW. Patients with neck pain demonstrate reduced electromyographic activity of the deep cervical flexor muscles during performance of the craniocervical flexion test. Spine. 2004;29:2108-14.

5. Lynch SS, Thigpen CA, Mihalik JP, Prentice WE, Padua D. The effects of an exercise intervention on forward head and rounded shoulder postures in elite swimmers. Br J Sports Med. 2010;44:376-81.

6. Dolan P, Adams M, Mutton W. Commonly adopted postures and their effect on the lumbar spine. Spine. 1988;13:197-201.

7. Griegel-Morris $\mathrm{P}$, Larson K, Mueller-Klaus K, Oatis CA. Incidence of common postural abnormalities in the cervical, shoulder, and thoracic regions and their association with pain in two age groups of healthy subjects. Phys Ther. 1992;72:425-31.

8. Haughie LJ, Fiebert IM, Roach KE. Relationship of forward head posture and cervical backward bending to neck pain. J Man Manip Ther. 1995;3:91-7.

9. Singla D, Veqar Z. Association between forward head, rounded shoulders, and increased thoracic kyphosis: a review of the literature. J Chiropr Med. 2017;16:220-9.

10. Kwon JW, Son SM, Lee NK. Changes in upper-extremity muscle activities due to head position in subjects with a forward head posture and rounded shoulders. J Phys Ther Sci. 2015;27:1739-42.

11. Thigpen CA, Padua DA, Michener LA, Guskiewicz K, Giuliani C, Keener JD, et al. Head and shoulder posture affect scapular mechanics and muscle activity in overhead tasks. Clin Rehabil. 2010;20:701-9.

12. Greenfield B, Catlin PA, Coats PW, Green E, McDonald JJ, North C. Posture in patients with shoulder overuse injuries and healthy individuals. J Orthop Sports Phys Ther. 1995;21:287-95.

13. Von Elm E, Altman DG, Egger M, Pocock SJ, Gøtzsche PC, Vandenbroucke J. The Strengthening the Reporting of Observational Studies in Epidemiology (STROBE) statement: guidelines for reporting ob- servational studies. Ann Intern Med. 2007;147:573-7.

14. Khosravi F, Rahnama L, Karimi N, Amiri M. The Correlation between Craniovertebral Angle and Scapular Dyskinesis in Adults. J Babol Univ Medical Sci. 2019;21:34-8.

15. Nemmers TM, Miller JW, Hartman MD. Variability of the forward head posture in healthy community-dwelling older women. J Geriatr Phys Ther. 2009;32:10-4.

16. Bovim G, Schrader H, Sand T. Neck pain in the general population. Spine. 1994;19:1307-9.

17. Hung M, Saltzman CL, Voss MW, Bounsanga J, Kendall R, Spiker R, et al. Responsiveness of the patient-reported outcomes measurement information system (PROMIS), neck disability index (NDI) and oswestry disability index (ODI) instruments in patients with spinal disorders. Spine J. 2019;19:34-40.

18. Kitis A, Celik E, Aslan UB, Zencir M. DASH questionnaire for the analysis of musculoskeletal symptoms in industry workers: a validity and reliability study. Appl Ergon. 2009;40:251-5.

19. Lee J-Y, Lim J-Y, Oh JH, Ko Y-M. Cross-cultural adaptation and clinical evaluation of a Korean version of the disabilities of arm, shoulder, and hand outcome questionnaire (K-DASH). J Shoulder Elbow Surg. 2008;17:570-4.

20. Kim CG, Lee ES. The Changes of in Headache due to Postural Improvement in Patients with Tension Headache with Forward Head Posture. J Kor Soi Neurotherapy. 2016;20:27-32.

21. Motialla T, Haghighi FM, Ghanbari A, Moezi SA, Saadat Z. The correlation between forward head posture and trigger points in trapezius muscle in subjects with chronic neck pain. J Res Rehabil Sci. 2013;8:989-97.

22. Nejati P, Lotfian S, Moezy A, Nejati M. The study of correlation between forward head posture and neck pain in Iranian office workers. Int J Occup Med Environ Health. 2015;28.

23. Lee K-J, Han H-Y, Cheon S-H, Park S-H, Yong $\mathrm{M}-\mathrm{S}$. The effect of forward head posture on muscle activity during neck protraction and retraction. J Phys Ther Sci. 2015;27:977-9. 